\title{
Design and synthesis of a zero-photoelastic birefringence polymer with a high glass-transition temperature by a random copolymerization method
}

\begin{abstract}
Houran Shafiee ${ }^{1,2}$, Akihiro Tagaya ${ }^{1,2}$ and Yasuhiro Koike ${ }^{2}$
We designed and synthesized a zero-photoelastic birefringence polymer that had a high glass-transition temperature $\left(T_{\mathrm{g}}\right)$ by introducing trans-stilbene units into the side chains of the copolymer by a random copolymerization method. The concentration of the trans-stilbene units required to compensate for photoelastic birefringence in the random copolymer was lower than that required in a polymer prepared by an anisotropic molecule dopant method, because the presence of an anisotropic moiety in the side chain of the polymer has a greater effect than an anisotropic molecule doped in the polymer. Furthermore, the polymer with trans-stilbene units in side chains showed a higher $T_{\mathrm{g}}$ than the polymer containing an anisotropic dopant. We conclude that the introduction of an anisotropic molecule of a similar or larger size to trans-stilbene into the side chain gives a polymer with a high $T_{\mathrm{g}}$. Polymer Journal (2011) 43, 306-312; doi:10.1038/pj.2010.136; published online 12 January 2011
\end{abstract}

Keywords: anisotropic molecule dopant method; glass-transition temperature; photoelastic birefringence; polarizability anisotropy; random copolymerization method; trans-stilbene methacrylate

\section{INTRODUCTION}

Polymeric materials are currently used in a variety of optical devices, such as optical films for liquid crystal displays (LCDs) ${ }^{1-3}$ optical disks ${ }^{4}$ and lenses. ${ }^{5}$ Display devices are key infrastructural elements in multimedia systems. As one of several kinds of flat-panel display that are available, LCDs are widely used in a range of applications, including computer monitors, televisions, instrument panels, mobile telephones and aircraft cockpit displays. In comparison with glass, polymers have the advantages of being more easily processed, more easily handled, lower in cost and lighter in weight. However, polymeric materials tend to exhibit birefringence, which restricts their use in optical devices that handle polarized light. Birefringence occurs when a polymer is elastically deformed below its glass-transition temperature $\left(T_{\mathrm{g}}\right)$, that is when it is in an elastic deformation region. This type of birefringence has been named as 'photoelastic birefringence.' It has been confirmed that photoelastic birefringence depends mainly on the orientation of the side chains of the polymer, whereas the main chains have little effect. ${ }^{6}$ When a polarizing plate in an LCD contracts, stress is applied to the polymer film causing birefringence. This photoelastic birefringence results in light leakage when the LCD is in its 'black' state, thereby impairing the quality of the image, particularly its contrast. A polymer that does not generate photoelastic birefringence, even when stressed during use of an LCD, and which thereby prevents light leakage is therefore desirable. Photoelastic birefringence is also observed in phase-difference films, which are used to increase the viewing angles of LCDs. Koike and co-workers proposed two methods for compensating for this birefringence (Figure 1): the anisotropic molecule dopant method $^{7-9}$ and the random copolymerization method. ${ }^{10-13}$

In the anisotropic molecule dopant method, the polymer is doped with a molecule that has an anisotropic polarizability and a rod-like form. The anisotropic dopant molecule must have an anisotropic form, such as a rod-like shape, so that it is oriented when the polymer is under stress, and it must show anisotropy in its polarizability to exhibit a birefringence that is opposite in direction of that of the polymer matrix. Generally, this method has the advantage of easy processing, as birefringence can be eliminated simply by doping the polymer with anisotropically polarizable molecule. However, doping with an anisotropic molecule results in a decrease in the $T_{\mathrm{g}}$ of the polymer matrix. ${ }^{8}$

In the random copolymerization method, monomers with negative and positive birefringences, which form polymers with negative and positive birefringence, respectively, are randomly copolymerized. As a result, the birefringence is canceled out when the monomers are present in a specific ratio. The resulting polymer, which is known as a 'zero-photoelastic birefringence polymer' exhibits no photoelastic birefringence under stress. ${ }^{11}$ On example of such a polymer is a 92:8 (w/w) copolymer of methyl methacrylate (MMA) and benzyl

${ }^{1}$ Integrated Design Engineering, Faculty of Science and Technology, Keio University, Yokohama, Japan and ${ }^{2}$ Koike Photonics Polymer Project, ERATO-SORST, Japan Science and Technology Agency, Kawasaki, Japan

Correspondence: H Shafiee, Koike Photonics Polymer Project, ERATO-SORST, Japan Science and Technology Agency, E-building, Shin-Kawasaki Town Campus, 7-1 Shin-Kawasaki, Saiwai-ku, Kawasaki 212-0032, Japan.

E-mail: houran@hotmail.com

Received 20 October 2010; revised and accepted 27 November 2010; published online 12 January 2011 
a

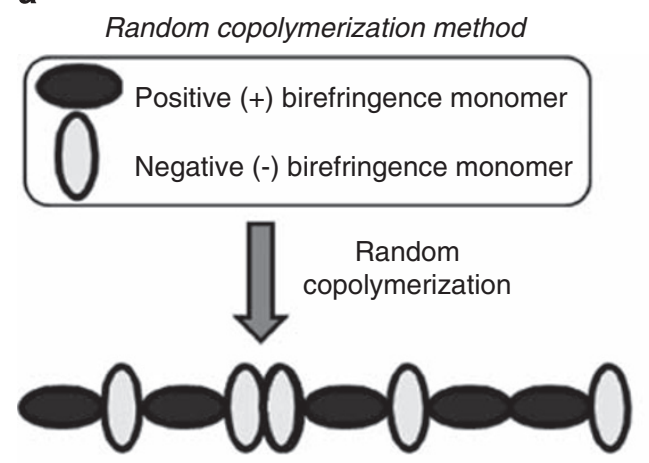

Zero-birefringence copolymer b Anisotropic molecule dopant method

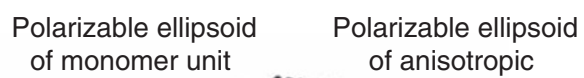

of monomer unit

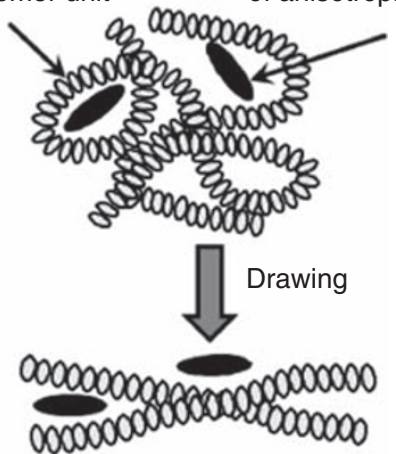

Figure 1 Methods for compensating for birefringence of polymers. (a) Anisotropic molecule dopant method; (b) random copolymerization method.

a

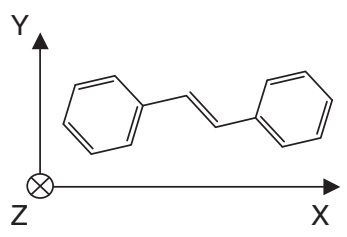

trans-Stilbene

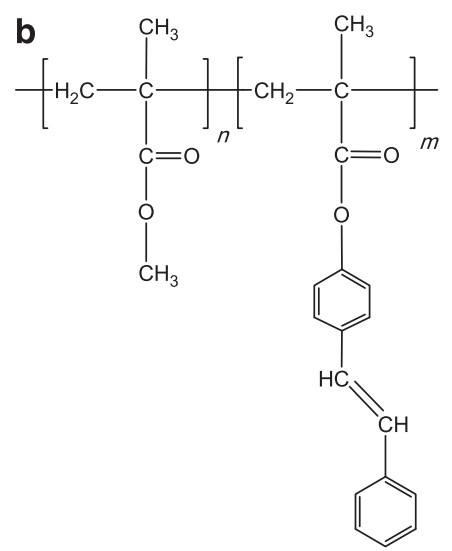

Poly(MMA-co-TSMA)

Figure 2 Molecular structures of (a) trans-stilbene and (b) poly(methyl methacrylate-co-trans-stilbene-4-yl methacrylate) (poly(MMA-co-TSMA)). MMA, methyl methacrylate; TSMA, trans-stilben-4-yl methacrylate.

methacrylate; however, the $T_{\mathrm{g}}$ of this polymer is $104{ }^{\circ} \mathrm{C}$, which is lower than that of poly(methyl methacrylate) (PMMA) $\left(T_{\mathrm{g}}: 109^{\circ} \mathrm{C}\right) .{ }^{13}$

Polymers that do not exhibit photoelastic birefringence during stress loading and which have a high $T_{\mathrm{g}}$ are required in many applications, for example, as polymer films for LCDs. ${ }^{14,15}$ The ideal monomer would be one that could be copolymerized with MMA, that would compensate for its photoelastic birefringence at a low concentration and that would produce a copolymer with a high $T_{\mathrm{g}}$, because MMA is a mass-produced, commercially available monomer with low cost that demonstrated high-light transmittance and provides excellent resistance to both chemical and weather corrosions. ${ }^{16}$ Consequently, it is necessary to design a monomer that has a highly anisotropic polarizability.

We therefore designed and synthesized a zero-photoelastic birefringence polymer with a high $T_{\mathrm{g}}$ by incorporating a trans-stilbene unit in the side chain to give a monomer with the high polarizability anisotropy. We compared the effects of the trans-stilbene unit in compensating for photoelastic birefringence by the random copolymerization method and by the anisotropic molecular dopant method. We have also clarified the mechanism by which the trans-stilbene unit compensates for photoelastic birefringence. Moreover, we compared

the $T_{\mathrm{g}}$ values for of zero-photoelastic birefringence polymers produced by these two methods, and we clarified the effects on $T_{\mathrm{g}}$ of the presence of trans-stilbene unit in the side chain. In the anisotropic molecule dopant method, trans-stilbene $\left(1,1^{\prime}\right.$-(E)-ethene-1,2-diyldibenzene; Figure 2a) was chosen as an anisotropic molecule and was doped into PMMA. In the random copolymerization method, trans-stilben-4-yl methacrylate (TSMA; 4-((E)-2-phenylvinyl)phenyl 2-methylacrylate; Figure 2b), which contains a trans-stilbene unit in the side chain, was polymerized with MMA.

\section{EXPERIMENTAL PROCEDURE \\ Preparation of TSMA monomer}

TSMA was prepared by treating trans-4-hydroxystilbene (4-((E)-2-phenylvinyl)phenol) with 2-methacryloyl chloride in the presence of triethylamine in diethyl ether. ${ }^{17,18}$

\section{Trans-4-hydroxystilbene}

A 1-1 round-bottomed flask was charged with a mixture of 4-hydroxybenzaldehyde $(122 \mathrm{~g}, 1 \mathrm{~mol})$, phenylacetic acid $(184 \mathrm{~g}, 1.35 \mathrm{~mol})$ and piperidine $(400 \mathrm{ml})$, and the mixture was refluxed for $6 \mathrm{~h}$. The mixture was then cooled and poured onto crushed ice. The mixture was acidified to $\mathrm{pH} \approx 5$ with concentrated hydrochloric acid diluted to $1: 6(\mathrm{v} / \mathrm{v})$ with water to dissolve the piperidine, and the suspended product was intensively stirred to break up larger particles. The crude product was filtered off, washed successively with dilute hydrochloric acid and water, dried and dissolved in ethyl acetate. The solution was then dried over sodium sulfate and concentrated on a rotary evaporator. The residue was vacuum distilled and the resulting product was further purified by column chromatography (Silicagel (60-120 mesh), ethyl acetate-hexane (1:5 $\mathrm{v} / \mathrm{v})$ ) to give pure trans-4-hydroxystilbene as a white solid; yield: $74.5 \mathrm{~g}(38 \%)$; m.p. $186-187^{\circ} \mathrm{C} .{ }^{1} \mathrm{H} \mathrm{NMR}\left(300 \mathrm{MHz}, \mathrm{CDCl}_{3}\right): \delta_{\mathrm{H}}=7.63(\mathrm{~d}, 2 \mathrm{H}), 7.46(\mathrm{~d}, 2 \mathrm{H})$, $7.40(\mathrm{~d}, 2 \mathrm{H}), 7.22(\mathrm{t}, 1 \mathrm{H}), 7.21(\mathrm{~d}, 2 \mathrm{H}), 7.01(\mathrm{~d}, 1 \mathrm{H}), 6.88(\mathrm{~d}, 1 \mathrm{H})$. Anal. calcd for $\mathrm{C}_{14} \mathrm{H}_{12} \mathrm{O}$ : C, 85.73; H, 6.17. Found: C, 85.67; H, 6.25.

\section{Trans-stilbene methacrylate}

Triethylamine $(30.3 \mathrm{~g}, 0.3 \mathrm{~mol})$ was added to a solution of trans-4-hydroxystilbene $(39.2 \mathrm{~g}, 0.2 \mathrm{~mol})$ in diethyl ether $(500 \mathrm{ml})$. A solution of methacryloyl chloride $(26 \mathrm{~g}, 0.25 \mathrm{~mol})$ in diethyl ether $(150 \mathrm{ml})$ was then added dropwise with stirring at room temperature. The mixture was then stirred for $5 \mathrm{~h}$, triethylamine hydrochloride was filtered off and the filtrate was washed successively with dilute hydrochloric acid ( $5 \mathrm{wt} \%)$, aqueous sodium carbonate $(5 \mathrm{wt} \%)$ and water. The organic phase was dried over anhydrous sodium sulfate. After removal of the sodium sulfate by filtration, the diethyl ether was evaporated on a rotary evaporator and the crude product was eluted with from silica gel (600-120 mesh) by using ethyl acetate-hexane (1:5 v/v). Recrystallization 
from hexane gave a pure white product; yield: $36.3 \mathrm{~g}(68.5 \%)$; m.p. $157^{\circ} \mathrm{C} .{ }^{1} \mathrm{H}$ $\operatorname{NMR}\left(300 \mathrm{MHz}, \mathrm{CDCl}_{3}\right): \delta_{\mathrm{H}}=7.64(\mathrm{~d}, 2 \mathrm{H}), 7.46(\mathrm{~d}, 2 \mathrm{H}), 7.40(\mathrm{~d}, 2 \mathrm{H}), 7.22$ $(\mathrm{t}, 1 \mathrm{H}), 7.20(\mathrm{~d}, 2 \mathrm{H}), 7.01(\mathrm{~d}, 1 \mathrm{H}), 6.88(\mathrm{~d}, 1 \mathrm{H}), 6.30(\mathrm{~d}, 1 \mathrm{H}), 5.92(\mathrm{~d}, 1 \mathrm{H}), 2.03$ (s, 3H). Anal. calcd for $\mathrm{C}_{18} \mathrm{H}_{16} \mathrm{O}_{2}: \mathrm{C}, 81.23 ; \mathrm{H}, 6.40$. Found: $\mathrm{C}, 81.41 ; \mathrm{H}, 6.37$.

\section{Preparation of polymer samples}

Preparation of poly(MMA-co-TSMA) by random copolymerization. MMA (99.8\%, Mitsubishi Gas Chemical Co., Tokyo, Japan) was distilled at $46-47^{\circ} \mathrm{C}$ and $13.3 \mathrm{kPa}$ to eliminate inhibitors and impurities. Copolymers with various weight ratios of MMA and TSMA were synthesized by solution polymerization in toluene at $90^{\circ} \mathrm{C}$ under an inert atmosphere of nitrogen by using $0.15 \mathrm{wt} \%$ (on the weight of the monomer) of tert-butyl 2-ethylhexaneperoxoate (98\%, NOF Corp., Tokyo, Japan) as an initiator. All polymers were precipitated in methanol for purification and dried at $90^{\circ} \mathrm{C}$ for $48 \mathrm{~h}$ at reduced pressure $(<1.33 \mathrm{~Pa})$. To determine the reactivity ratio of the monomers experimentally, we needed to know the copolymer composition. The purified samples were dissolved in chloroform and analyzed by using ${ }^{1} \mathrm{H}$ NMR (JNM-LA400, JEOL Ltd, Tokyo, Japan). To investigate the infrared (IR) dichroism and birefringence of the polymers, films were prepared by dissolving poly(MMA-co-TSMA) in dichloromethane and spreading the polymer solution onto a glass plate with a knife coater. The sample films were dried at room temperature for $4 \mathrm{~h}$ and then at $90^{\circ} \mathrm{C}$ under reduced pressure for $24 \mathrm{~h}$ to eliminate the solvent. The films were then cut into dumbbell-shaped specimens.

Preparation of PMMA doped with trans-stilbene by the anisotropic molecule dopant method. MMA (99.8\%, Mitsubishi Gas Chemical Co.) was distilled at $46-47^{\circ} \mathrm{C}$ and $13.3 \mathrm{kPa}$ to eliminate inhibitors and impurities. A mixture of tertbutyl 2-ethylhexaneperoxoate (98\%, NOF Corp.) as an initiator and butane-1thiol (95\%, Wako Pure Chemical Industries Ltd, Osaka, Japan) as a chaintransfer agent was added to the MMA in amounts of $0.4 \mathrm{wt} \%$ and $0.1 \mathrm{wt} \%$, respectively. The mixtures were injected into cylindrical glass tubes and then polymerized at $70^{\circ} \mathrm{C}$ for $24 \mathrm{~h}$. The resulting bulk polymer samples were heat treated at $90^{\circ} \mathrm{C}$ for $24 \mathrm{~h}$. The polymer was precipitated in methanol for purification and dried at $90^{\circ} \mathrm{C}$ for $48 \mathrm{~h}$ at reduced pressure $(<1.33 \mathrm{~Pa})$. To prepare film samples, mixtures of PMMA and trans-stilbene (98\%, Wako Pure Chemical Industries Ltd) were dissolved in dichloromethane, and the solution was spread onto a glass plate with a knife coater. The sample films were dried at room temperature for $4 \mathrm{~h}$ and then at $90^{\circ} \mathrm{C}$ at reduced pressure for $24 \mathrm{~h}$ to eliminate the solvent. The films were finally cut into dumbbell-shaped specimens.

\section{Measurement of the photoelastic birefringence}

The photoelastic birefringence $\left(\Delta n_{\mathrm{ph}}\right)$ generated in a stressed material is defined as follows:

$$
\Delta n_{\mathrm{ph}}=C \cdot \sigma
$$

where $C$ is the photoelastic coefficient of the material and $\sigma$ is the stress. ${ }^{19}$ The sign of photoelastic birefringence is defined as $\Delta n=n_{\|}-n_{\perp}$, where $n_{\|}$and $n_{\perp}$ are the refractive indices for light polarized in the directions parallel and perpendicular, respectively, to the direction of drawing (tensile stress). The shape of a typical sample is shown in Figure 3a and the experimental setup is shown in Figure 3b. The photoelastic birefringence of each film sample was measured at various levels of uniaxial tensile stress (about 1\% strain) at room temperature by optical heterodyne interferometry at a wavelength of $633 \mathrm{~nm}$ using a birefringence measurement system (ABR-10, UNIOPT Corporation, Ltd., Shizuoka, Japan). ${ }^{20}$

\section{IR measurements}

To analyze the motion of the polymer molecules during the generation of photoelastic birefringence, we measured the IR dichroism of the polymer film in an elastically deformed state. ${ }^{9}$ The experimental setup is shown in Figure 4. The sample film was fixed and then uniaxially drawn at room temperature by using actuators. The absorbance of the drawn film was measured at $400-4000 \mathrm{~cm}^{-1}$ by using a Fourier-transform IR spectrometer (7000e FT-IR, Varian Inc., Santa Clara, CA, USA). We measured the absorbance of IR light polarized in the directions parallel and perpendicular to the drawing direction. We selected a wavelength

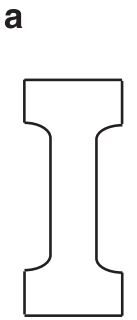

Film sample b

Birefringenece meaurement system

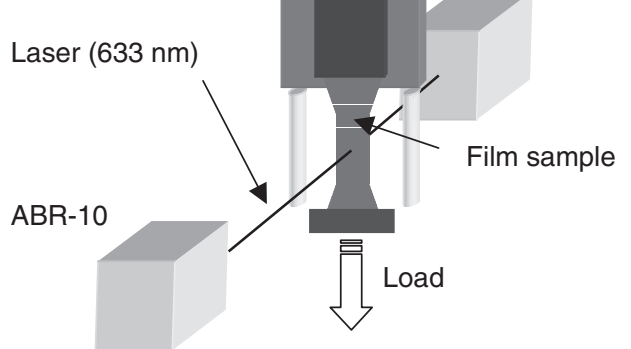

Figure 3 Schematic representation of the system for measuring photoelastic birefringence. (a) Film sample. (b) Birefringence measurement system.

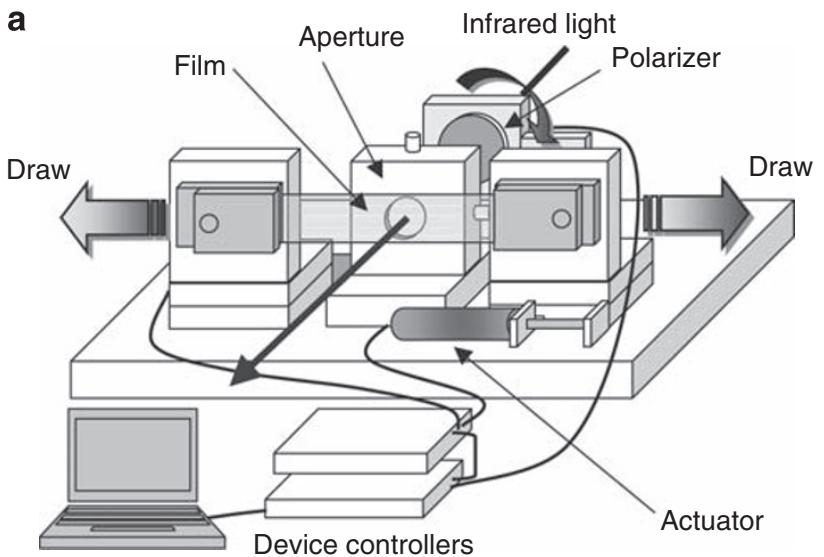

Measurement equipment for the analysis of IR dichroism

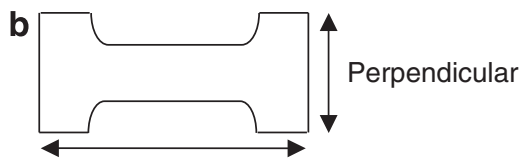

Parallel

Film sample

Figure 4 Schematic representation of equipment for the measurement of the IR dichroism of elastically deformed polymer films. (a) Measurement equipment for the analysis of infrared dichroism. (b) Film sample.

of $696 \mathrm{~cm}^{-1}$ for observing the ring out-of-plane deformation of the benzene rings of trans-stilbene ${ }^{21-24}$ in PMMA doped with trans-stilbene. In the case of poly(MMA-co-TSMA), we selected a wavelength of $693 \mathrm{~cm}^{-1}$ to observe the ring out-of-plane deformations of the monosubstituted and 1,4-disubstituted benzene rings of TSMA. ${ }^{25-27}$ The dichroic ratio $D$ is defined as $D=A_{\|} / A_{\perp}$, where $A_{\|}$and $A_{\perp}$ are the absorbances for linearly polarized IR light in the directions parallel and perpendicular to the drawing direction, respectively. The orientation function $f$ is related to the dichroic ratio $D$ by the following equation:

$$
f=\frac{D-1}{D+2} \cdot \frac{2 \cot ^{2} \alpha+2}{2 \cot ^{2} \alpha-1}
$$

where $\alpha$ is the angle between the transition moment vector of the absorbing group and the long axis of the molecule. ${ }^{28,29}$ We assumed that $\alpha=0$, because the trans-stilbene is a planar rod-like molecule and the transition moment vector of the absorbing group is perpendicular to the plane of the benzene rings of trans-stilbene. 


\section{Other measurements}

The stress-strain curves of the sample films were investigated at room temperature to confirm that they exhibited elastic behavior. The stress-strain curves of the samples were measured at a strain rate of $0.5 \mathrm{~mm} \mathrm{~min}^{-1}$ by using a universal tensile testing machine (Tensilon RTC-1210A, A\&D Co., Ltd, Tokyo, Japan) at room temperature.

The values of the glass-transition temperature $\left(T_{\mathrm{g}}\right)$ of the purified polymers were measured by differential scanning calorimetry (DSC-60, Shimadzu Corp., Kyoto, Japan). All polymer samples were heated from room temperature to around $160{ }^{\circ} \mathrm{C}$ in air, then cooled to $-20^{\circ} \mathrm{C}$ and reheated to $160{ }^{\circ} \mathrm{C}$ at a scanning rate of $10^{\circ} \mathrm{C} \mathrm{min}^{-1}$. The $T_{\mathrm{g}}$ was measured during the second heating scan.

The polarizability was calculated by means of a semi-empirical molecular orbital calculation program (SCIGRESS, Fujitsu Ltd., Kanagawa, Japan). The moderate neglect of differential overlap (MNDO Hamiltonian) was adopted with AM1. ${ }^{30,31}$ First, the molecular geometry was optimized, and then the electronic polarizability was calculated. The polarizability anisotropy $\Delta \alpha$ was calculated from the following equation:

$$
\Delta \alpha=\alpha_{\mathrm{XX}}-\left(\alpha_{\mathrm{YY}}+\alpha_{\mathrm{ZZ}}\right) / 2
$$

where $\alpha_{X X}, \alpha_{Y Y}$ and $\alpha_{Z Z}$ are the polarizabilities along the $X, Y$ and $Z$ axes, respectively. ${ }^{7}$ The $X, Y$ and $Z$ axes are aligned to the principal axes of polarizability tensor.

\section{RESULTS}

The photoelastic coefficients and glass-transition temperatures of the polymers

Figure $5 \mathrm{a}$ is a plot of the photoelastic birefringence of the poly(MMAco-TSMA) samples against the stress, and Figure $5 \mathrm{~b}$ is a similar plot for PMMA samples doped with trans-stilbene. The photoelastic birefringences of poly(MMA-co-TSMA) and of PMMA doped with transstilbene decreased and shifted from the negative side to the positive side as the concentration of trans-stilbene moieties increased. The slope of a straight-line approximation corresponds to the photoelastic coefficient $C$ of the samples. Figure 6 shows plots of the photoelastic coefficients of poly(MMA-co-TSMA) and of PMMA doped with trans-stilbene against the concentration of trans-stilbene moieties (mol\%). We calculated the concentration of trans-stilbene unit that is required to eliminate the photoelastic coefficient. As Table 1 shows, the photoelastic coefficient of poly(MMA-co-TSMA) became zero on introducing $0.8 \mathrm{~mol} \%$ of trans-stilbene moieties, and that of PMMA doped with trans-stilbene became zero on addition of $1.7 \mathrm{~mol} \%$ of trans-stilbene. The $T_{\mathrm{g}}$ of polymers of these compositions is shown in

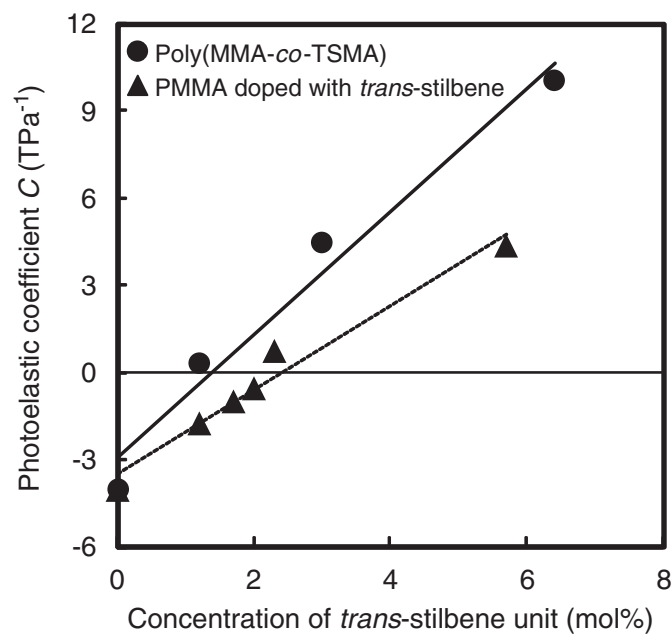

Figure 6 Plots of the photoelastic coefficients of poly(MMA-co-TSMA) and of poly(methyl methacrylate) doped with trans-stilbene against the concentration of trans-stilbene units (mol\%). MMA, methyl methacrylate; TSMA, trans-stilben-4-yl methacrylate.

Table 1 Concentration of the trans-stilbene units in the PMMA doped with trans-stilbene and in the poly(MMA-co-TSMA) required to eliminate the birefringence, and the glass-transition temperature of each polymer sample

\begin{tabular}{|c|c|c|}
\hline & $\begin{array}{c}\text { Zero-birefringence } \\
\text { composition }^{\mathrm{a}}(\mathrm{mol} \%)\end{array}$ & $\begin{array}{l}\text { Glass-transition } \\
\text { temperature }\left(\mathrm{T}_{\sigma}\right)^{\circ} \mathrm{C}\end{array}$ \\
\hline
\end{tabular}

\begin{tabular}{lcr}
\hline Poly(MMA-co-TSMA) & 0.8 & 125 \\
PMMA+trans-stilbene & 1.7 & 84 \\
PMMA & - & 109
\end{tabular}

Abbreviations: MMA, methyl methacrylate; PMMA, poly(methyl methacrylate); TSMA, transstilben-4-yl methacrylate.

amol\%: molar ratio of trans-stilbene to methyl methacrylate.

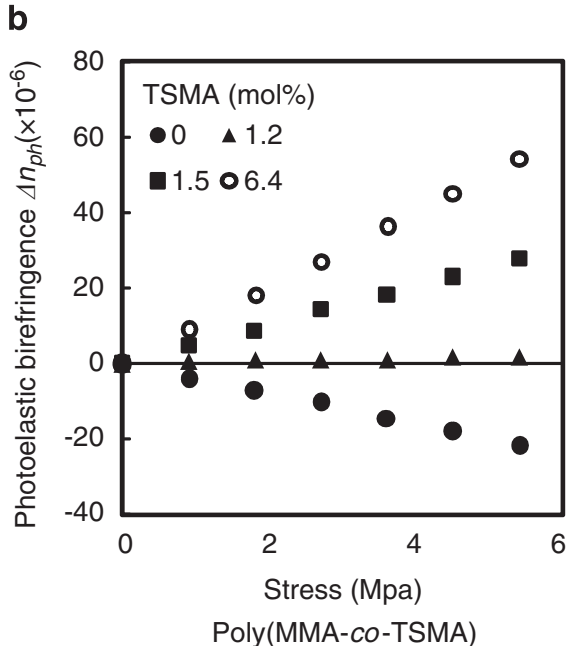

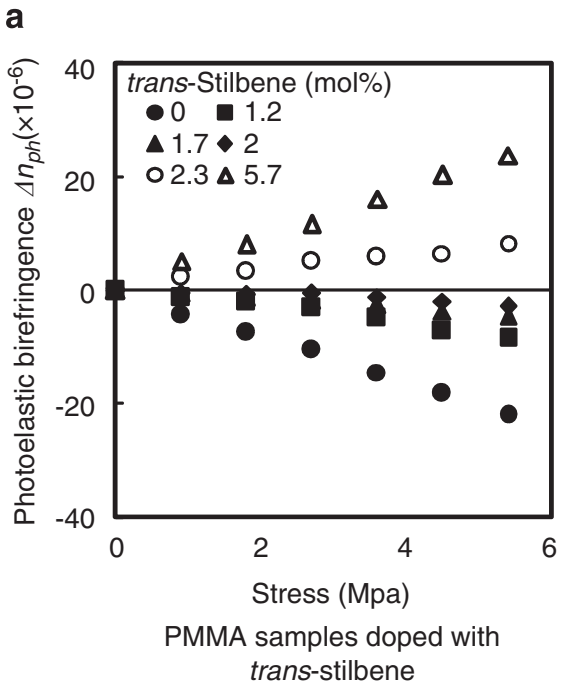

Figure 5 Plots of the photoelastic birefringence of the samples at a wavelength of $633 \mathrm{~nm}$ against the stress for (a) poly(MMA-co-TSMA) and (b) poly(methyl methacrylate) samples doped with trans-stilbene. 


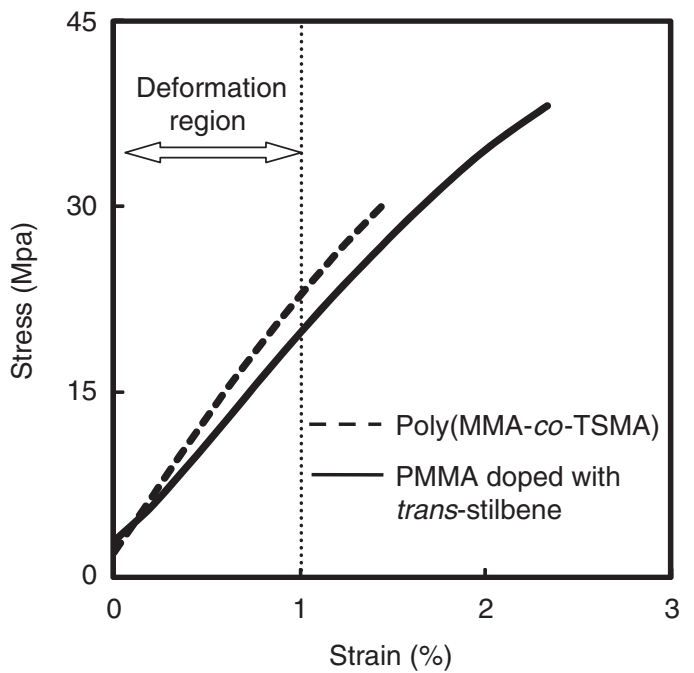

Figure 7 Stress-strain curves of films of poly(MMA-co-TSMA) and of poly(methyl methacrylate) doped with trans-stilbene. The thickness of the films was $10 \mu \mathrm{m}$. MMA, methyl methacrylate; TSMA, trans-stilben-4-yl methacrylate.

Table 1. The $T_{\mathrm{g}}$ of PMMA doped with trans-stilbene $(1.7 \mathrm{~mol} \%)$ was $84^{\circ} \mathrm{C}$, whereas that of poly(MMA-co-TSMA) containing $0.8 \mathrm{~mol} \%$ of trans-stilbene moieties was $125^{\circ} \mathrm{C}$. These results show that the polymer that we obtained by introducing trans-stilbene units into the side chains by random copolymerization had a higher glass-transition temperature than the polymer that we prepared by doping with an anisotropic molecule.

\section{Analysis of the effects of trans-stilbene units in compensating} for photoelastic birefringence

The stress-strain curves of poly(MMA-co-TSMA) and PMMA doped with trans-stilbene films are shown in Figure 7. The thickness of the films was $10 \mu \mathrm{m}$ in both cases. We decided to draw the films uniaxially to an elongation ratio of $1 \%$ at room temperature to analyze the IR dichroism of the films, because the deformation of each film was almost elastic when the strain was within about $1 \%$, because of the linear curve of the film. The composition of the poly(MMA-coTSMA) copolymer was analyzed by means of ${ }^{1} \mathrm{H}$ NMR spectroscopy, and it was found that the concentration of trans-stilbene units was 6.4 mol\%. The composition of PMMA doped with trans-stilbene was similarly analyzed, and it was shown to contain $5.4 \mathrm{~mol} \%$ of transstilbene. The IR dichroism of the poly(MMA-co-TSMA) film and the trans-stilbene-doped PMMA film were measured to investigate the motions of the molecule during the generation of photoelastic birefringence by the application of tensile stress. Figure 8 shows the absorption of the ring out-of-plane deformation of the benzene rings of trans-stilbene in poly(MMA-co-TSMA) (a) before stress loading and (b) during stress loading. The solid lines show the IR absorption spectra in the direction perpendicular to the drawing direction, and the broken lines show the absorbance of the light polarized in the parallel direction. The thickness of the sample was $12 \mu \mathrm{m}$. As can be seen in Figure 8a, the ring out-of-plane absorbance $\left(693 \mathrm{~cm}^{-1}\right)$ in the parallel direction before stress loading is almost the same as that in the perpendicular direction. Figure $8 \mathrm{~b}$ shows that the absorbance of the ring out-of-plane in the perpendicular direction during stress loading is larger than that in the parallel direction, suggesting that the benzene rings of the trans-stilbene were oriented in the direction perpendicular to the drawing direction during stress loading. a

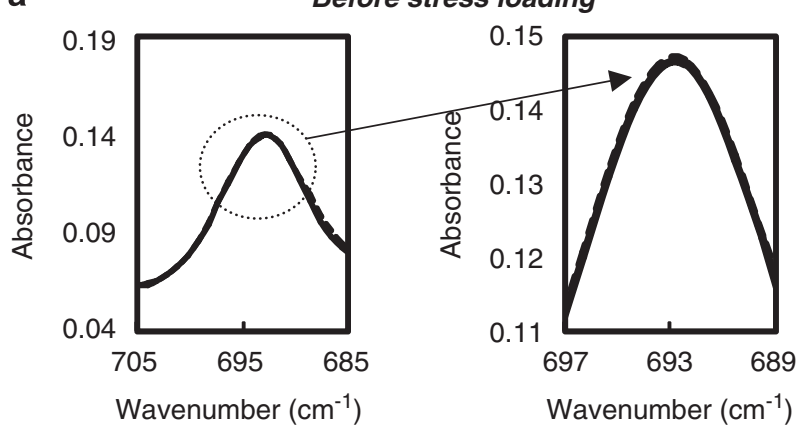

b

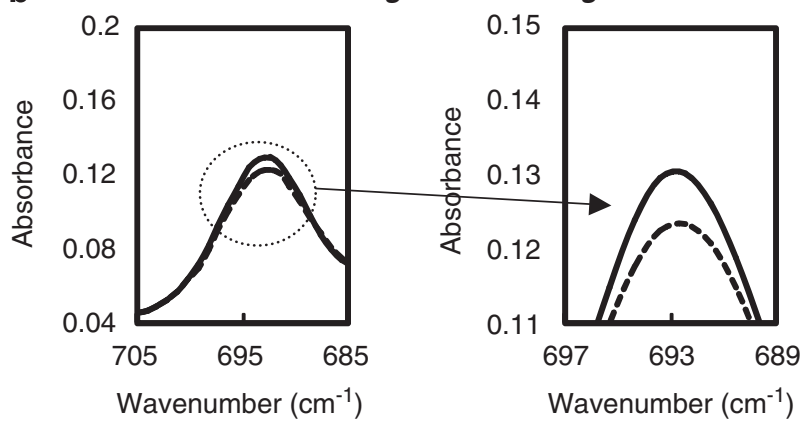

Figure 8 Infrared absorption spectra of a $12-\mu \mathrm{m}$-thick films of poly(MMA-coTSMA) (6.4 mol\% trans-stilbene) (a) before stress loading and (b) during stress loading. The out-of-plane deformation of the benzene ring of transstilbene was observed at $693 \mathrm{~cm}^{-1}$. MMA, methyl methacrylate; TSMA, trans-stilben-4-yl methacrylate.

Table 2 Dichroic ratio $(D)$ and orientation function $(f)$ of the out-ofplane deformation of the benzene ring of trans-stilbene before stress loading and during stress loading. $\Delta f$ is the difference between the orientation function before stress loading $\left(f_{\mathrm{B}}\right)$ and during stress loading $\left(f_{\mathrm{D}}\right)$. (a) Poly(MMA-co-TSMA) and (b) PMMA doped with trans-stilbene at a wavenumber of $696 \mathrm{~cm}^{-1}$

\begin{tabular}{|c|c|c|}
\hline & Before stress loading & During stress loading \\
\hline \multicolumn{3}{|c|}{ (a) Poly(MMA-co-TSMA) } \\
\hline$D$ & 1.004 & 0.936 \\
\hline$f$ & $f_{\mathrm{B}}:-0.002$ & $f_{D}: 0.043$ \\
\hline$\Delta f=f_{\mathrm{D}}-f_{\mathrm{B}}$ & \multicolumn{2}{|c|}{0.046} \\
\hline \multicolumn{3}{|c|}{ (b) PMMA doped with trans-stilbene } \\
\hline$D$ & 1.002 & 0.985 \\
\hline$f$ & $f_{\mathrm{B}}:-0.001$ & $f_{\mathrm{D}}: 0.010$ \\
\hline$\Delta f=f_{\mathrm{D}}-f_{\mathrm{B}}$ & \multicolumn{2}{|c|}{0.012} \\
\hline
\end{tabular}

Abbreviations: MMA, methyl methacrylate; PMMA, poly(methyl methacrylate); TSMA, transstilben-4-yl methacrylate.

Table 2 lists the dichroic ratio $D$ and the orientation function before stress loading $\left(f_{\mathrm{B}}\right)$ and during stress loading $\left(f_{\mathrm{D}}\right)$ of trans-stilbene in (a) poly(MMA-co-TSMA) and (b) PMMA doped with trans-stilbene, as calculated by using Equation (2). The value of $f_{\mathrm{B}}$ was almost zero before stress loading, showing that the trans-stilbene units in the undrawn film were not oriented. During stress loading, the value of $f_{\mathrm{D}}$ of the oriented film was more than zero, showing that the transstilbene units were oriented in the direction perpendicular to the draw direction. We therefore confirmed that the trans-stilbene units were 


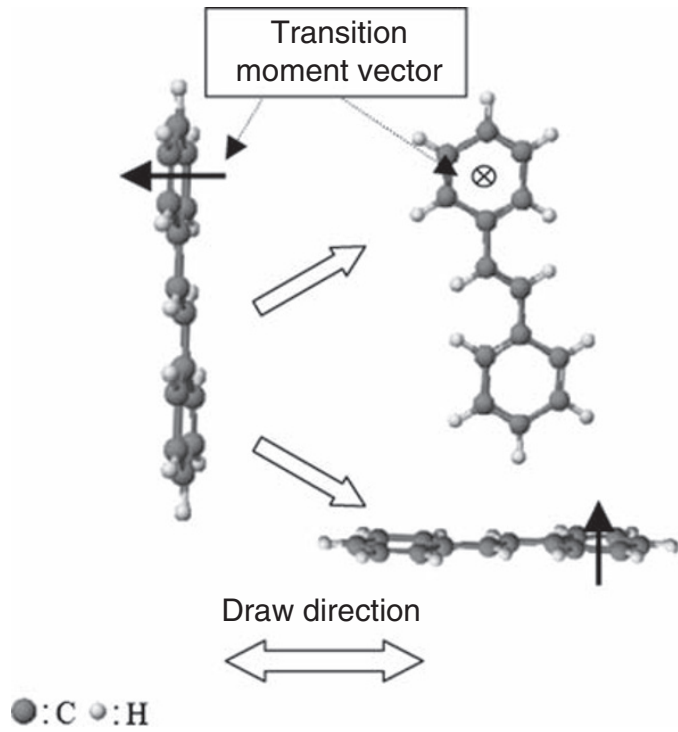

Figure 9 Estimated motion of trans-stilbene units in uniaxially stressed poly(methyl methacrylate). ${ }^{9}$

highly oriented during stress loading. As can be seen in Table 2b, the trans-stilbene molecules in PMMA are not oriented before stress loading and during stress loading they become oriented in the direction perpendicular to the draw direction. We have therefore confirmed that trans-stilbene was moved elastically by the load stress. The motion of trans-stilbene units in poly(MMA-co-TSMA) is virtually identical to that of trans-stilbene molecules in PMMA as described in ref. 9. The two types of motions of trans-stilbene unit were estimated, as shown in Figure 9. The transition moment vector is perpendicular to the benzene ring and the polarizability of the benzene ring is higher in the direction perpendicular to the transition moment vector. Both of the motions steer the transition moment vector in the direction perpendicular to the draw direction.

\section{DISCUSSION}

We found that the trans-stilbene moiety had an effect of 2.1 times greater in compensating for the photoelastic birefringence in poly (MMA-co-TSMA) than that in PMMA doped with trans-stilbene. The introduction of trans-stilbene unit into the side chains of the polymer therefore enhanced the compensatory effect in photoelastic birefringence.

In general, any factor that affects segmental mobility will affect the $T_{\mathrm{g}}$. This includes factors that influence the nature of the moving segment, chain stiffness or steric hindrance, as well as those that affect the free volume that is available for segmental motion. As a general rule, any structural feature that reduces chain mobility or free volume will increase the $T_{\mathrm{g} .}{ }^{32}$ It has been observed that the size and flexibility of side groups can alter the $T_{\mathrm{g}}$ of polymers. ${ }^{33,34}$ As a result, a high- $T_{\mathrm{g}}$ polymer was obtained by introducing trans-stilbene units into the side chain, because the trans-stilbene units inhibit the motions of the molecular chains and reduce chain mobility. We estimated that high$T_{\mathrm{g}}$ polymer could be obtained by introducing anisotropic molecule having a rod-like form of a size that is similar to or larger than that of trans-stilbene into the side chain.

Birefringence can be formulated as the sum of the polarizability anisotropy and the orientation function of all the functional groups of a repeating unit of the polymer as follows: ${ }^{6,28}$

$$
\Delta n=\Delta \alpha_{1} f_{1} \phi_{1}+\Delta \alpha_{2} f_{2} \phi_{2}+\ldots+\Delta \alpha_{i} f_{i} \phi_{i} .
$$

Table 3 Polarizability anisotropies of the $\mathrm{CH}_{2}=\mathrm{O}, \mathrm{CH}_{3} \mathrm{OCH}_{3}$ and trans-stilbene calculated by using SCIGRESS

\begin{tabular}{|c|c|c|c|c|}
\hline \multirow[b]{2}{*}{ Anisotropic unit } & \multicolumn{3}{|c|}{ Polarizability $\propto(A U)$} & \multirow[b]{2}{*}{ Polarizability anisotropy $\Delta \alpha(A U)$} \\
\hline & $\alpha_{x x}$ & $\alpha_{Y Y}$ & $\alpha_{\mathrm{ZZ}}$ & \\
\hline $\mathrm{CH}_{2}=\mathrm{O}$ & 18.68 & 10.60 & 2.46 & 12.15 \\
\hline $\mathrm{CH}_{3} \mathrm{OCH}_{3}$ & 26.40 & 19.54 & 18.16 & 7.55 \\
\hline trans-Stilbene & 260.59 & 142.32 & 20.52 & 179.17 \\
\hline
\end{tabular}

Here, $\Delta \alpha_{i}, f_{i}$ and $\phi_{i}$ are the polarizability anisotropy, the orientation function and the volume fraction of the ith functional group, respectively. It follows that functional groups with a high polarizability anisotropy will have a major effect on the birefringence. In poly (MMA-co-TSMA) and PMMA doped with trans-stilbene, the polarizability anisotropies of the $\mathrm{C}=\mathrm{O}, \mathrm{C}-\mathrm{O}-\mathrm{C}$ and trans-stilbene units are higher than those of other functional groups. ${ }^{6,8}$ The polarizability anisotropies of the $\mathrm{C}=\mathrm{O}, \mathrm{C}-\mathrm{O}-\mathrm{C}$ and trans-stilbene units were calculated by means of SCIGRESS (Table 3). However, it is difficult to calculate the polarizability anisotropy or polarizability tensor of a partial structure of molecule $(\mathrm{C}=\mathrm{O}$ and $\mathrm{C}-\mathrm{O}-\mathrm{C})$. We consider a small molecule that is roughly equivalent to the side chain structure. Actually, we used the following molecule structure; $\mathrm{CH}_{2}=\mathrm{O}$ instead of $\mathrm{C}=\mathrm{O}$ bond and $\mathrm{CH}_{3} \mathrm{OCH}_{3}$ instead of $\mathrm{C}-\mathrm{O}-\mathrm{C}$ bond for calculating the polarizability anisotropy of $\mathrm{C}=\mathrm{O}$ and $\mathrm{C}-\mathrm{O}-\mathrm{C}$. Then, the polarizability anisotropy of the small molecule was calculated. The calculations are performed by using the MO-G method using the finite difference technique by SCIGRESS. In the case of trans-stilbene, the polarizability anisotropy of trans-stilbene molecule was calculated, because it is difficult to calculate the trans-stilbene unit in the side chain of TSMA. We calculated that the polarizability anisotropy of the trans-stilbene moiety is almost 17 times higher than those of the $\mathrm{C}=\mathrm{O}$ and $\mathrm{C}-\mathrm{O}-\mathrm{C}$ groups. This method is not perfect for calculating the polarizability anisotropy of a side chain of molecule, but we think it is valid for comparing the polarizability anisotropy of trans-stilbene and those of $\mathrm{C}=\mathrm{O}$ and $\mathrm{C}-\mathrm{O}-\mathrm{C}$. Consequently, even if the volume fraction of trans-stilbene moiety is low, the motion of trans-stilbene units has a major impact on the photoelastic birefringence. Because the transstilbene unit had a 2.1 times greater effect in eliminating photoelastic birefringence in poly(MMA-co-TSMA) than that in PMMA doped with trans-stilbene, we assumed that the degree of orientation of transstilbene units in the poly(MMA-co-TSMA) was higher than that in PMMA doped with trans-stilbene. The results confirmed the hypothesis that, because the concentration of trans-stilbene unit in the poly(MMA/TSMA) was a maximum of $6.4 \mathrm{~mol} \%$, nearly all the trans-stilbene units are located adjacent to side chains of MMA units in a poly(MMA-co-TSMA) chain. We deduced that the motions of MMA units of a poly(MMA-co-TSMA) chain during the generation of photoelastic birefringence is similar to that in a PMMA chain, because the majority of repeating units in the poly(MMA-co-TSMA) chain are MMA units. Photoelastic birefringence is generated in PMMA when the side chains of the PMMA molecules are oriented and the main chain is barely oriented during stress loading. ${ }^{6}$ We estimated that the intramolecular interaction between the side chains of the MMA units and the trans-stilbene units in the poly(MMA-coTSMA) is stronger than that between the PMMA chain and the transstilbene molecules in PMMA doped with trans-stilbene, because the trans-stilbene units of poly(MMA-co-TSMA) are thought to be closer to the main chain than are the trans-stilbene molecules in doped PMMA. 
This discussion suggests that not only trans-stilbene units, but also the other anisotropic molecule structures with a rod-like form and high polarizability anisotropy, such as diphenylacetylene, trans, trans1,4-diphenyl-1,3-butadiene or trans-chalcone ${ }^{8}$ units, in the side chain of the copolymer might have the same effect in compensating for photoelastic birefringence and producing a polymer with a high $T_{\mathrm{g}}$.

\section{CONCLUSIONS}

We have demonstrated that a copolymer with zero photoelastic birefringence and a high $T_{\mathrm{g}}$ can be realized by introducing an anisotropic molecular structure with a rod-like form and a high polarizability anisotropy $\Delta \alpha$ into the side chains of the polymer.

We found that the concentration of anisotropic molecule required to compensate for photoelastic birefringence in the random copolymerization method is lower than that required in the anisotropic molecule dopant method, because an anisotropic moiety in the side chain of a copolymer has a greater effect than does an anisotropic molecule doped in the polymer. In addition, by introducing transstilbene units into the side chain of a MMA copolymer, we obtained a polymer with a higher glass-transition temperature than that of PMMA doped with trans-stilbene. We conclude that a polymer with a high $T_{\mathrm{g}}$ can be obtained by introducing anisotropic moieties of a size similar to trans-stilbene or larger into the side chain.

\section{ACKNOWLEDGEMENTS}

We thank Dr Frantisek Mikes of the Polymer Research Institute of the Polytechnic University of New York for synthesizing the trans-stilbene methacrylate monomer.

1 Mori, H., Itoh, Y., Nishiura, Y., Nakamura, T. \& Shinagawa, Y. Performance of a novel optical compensation film based on negative birefringence of discotic compound for wide-viewing-angle twisted-nematic liquid-crystal displays. Jpn. J. Appl. Phys. 36, 143-147 (1997).

2 Kawamoto, H. The history of liquid-crystal displays. Proc. IEEE 90, 460-500 (2002).

3 Hatalis, K. M., Kanichi, J. \& Summers, J. C. Flat panel display materials 2. Mater. Res. Soc. Symp. Proc. 424, 295-298 (1997).

4 Bernacki, E. \& Mansuripur, M. Investigation of substrate birefringence effects on optical-disk performance. Appl. Opt. 32, 6547-6555 (1993).

5 Koike, Y., Tanio, N., Nihei, E. \& Ohtsuka, Y. Gradient-index polymer materials and their optical devices. Polym. Eng. Sci. 29, 1200-1204 (1989).

6 Shafiee, H., Tagaya, A. \& Koike, Y. Mechanism of generation of photoelastic birefringence in methacrylate polymers for optical devices. J. Polym. Sci. Part B Polym. Phys. 48, 2029-2037 (2010).

7 Tagaya, A., Iwata, S., Kawanami, E., Tsukahara, H. \& Koike, Y. Zero-birefringence polymer by the anisotropic molecule dope method. Appl. Opt. 40, 3677-3683 (2001).

8 Tagaya, A., Iwata, S., Kawanami, E., Tsukahara, H. \& Koike, Y. Anisotropic molecule dopant method for synthesizing a zero-birefringence polymer. Jpn. J. Appl. Phys. 40, 6117-6123 (2001).
9 Ohkita, H., Ishibashi, K., Tanaka, R., Tagaya, A. \& Koike, Y. Compensation of photoelastic birefringence of polymers by anisotropic molecules and analysis of compensation mechanism. Jpn. J. Appl. Phys. 44, 3975-3982 (2005).

10 Iwata, S., Tsukahara, Y., Nihei, E. \& Koike, Y. Compensation for birefringence of oriented polymers by random copolymerization method. Jpn. J. Appl. Phys. 35, 3896-3901 (1996).

11 Iwata, S., Tsukahara, Y., Nihei, E. \& Koike, Y. Transparent zero-birefringence copolymer and its optical properties. Appl. Opt. 36, 4549-4555 (1997).

12 Tagaya, A., Ohkita, H., Harada, T., Ishibashi, K. \& Koike, Y. Zero-birefringence optical polymers. Macromolecules 39, 3019-3023 (2006).

13 Tagaya, A., Harada, T. \& Koike, Y. Design of zero zero-birefringence polymers for injection molded products with low birefringence. Seikei-Kakou 21, 426-435 (2009).

14 Ohkita, H., Abe, Y., Kojima, H., Tagaya, A. \& Koike, Y. Birefringence reduction method for optical polymers by the orientation-inhibition effect of silica particles. Appl. Phys. Lett. 84, 3534-3536 (2004).

15 Hatano, T., Yamanaka, S. \& Arakawa, K. Polarizing plate, method for producing polarizing plate, and liquid crystal display device. US Patent 2010/0220267A1 (2010).

16 Brydson, J. A. Plastic Materials 376 (Butterworth Scientific: London, UK, 1982).

17 Tsai, F.- J., Torkelson, J. M. \& Lewis, F. D. Photoresponsive behavior of methyl methacrylate/trans-4-stilbene methacrylate statistical copolymers in solution and the solid state. Macromolecules 23, 1487-1493 (1990).

18 Güsten, H. \& Salzwedel, M. Die kernmagnetischen resonanzspektren substituierter trans-stilbene. Tetrahedron 23, 173-185 (1967).

19 Tsukiji, M., Kowa, H., Muraki, K., Umeda, N., Imoto, K., Kanasaki, M., Tahara, K., Morii, K. \& Tajitsu, Y. Measurement system for very small photoelastic constant of polymer films. Macromol. Symp. 242, 235-240 (2006).

20 Umeda, N. \& Kohwa, H. Measurement of the residual birefringence distribution in glass laser disk by transverse zeeman laser. Electron. Commun. Jpn. 74(Part 2), 21-28 (1991).

21 Dirlikov, S. K. \& Koenig, J. L. Assignment of the carbon-hydrogen stretching and bending vibrations of poly(methyl methacrylate) by selective deuteration. Appl. Spectrosc. 33, 555-561 (1979).

22 Nyquist, R. A. Interpreting Infrared, Raman, and Nuclear Magnetic Resonance Spectra Vol. 2, 351 (Academic Press, San Diego, CA, 2001).

23 Shimanouchi, T., Kakiuti, Y. \& Gamo, I. Out-of-plane $\mathrm{CH}$ vibrations of benzene derivatives. J. Chem. Phys. 25, 1245-1251 (1956).

24 Margoshes, M. \& Fassel, V. A. The infrared spectra of aromatic compounds: I. The outof-plane $\mathrm{C}-\mathrm{H}$ bending vibrations in the region $625-900 \mathrm{~cm}^{-1}$. Spectrochim. Acta 7 , 14-24 (1955).

25 Socrates, G. Infrared Characteristic Group Frequencies; Tables and Charts 2nd edn (Wiley, Chichester, 1994).

26 Higuchi, S., Tsuyama, H., Tanaka, S. \& Kamada, H. Some considerations on the out-ofplane vibration bands of $\mathrm{Ph}_{n} \mathrm{X}$ type molecules in the $800-670 \mathrm{~cm}^{-1}$ region in relation to the estimation of the twist angle $\theta$ of benzene rings from their intensities. Spectrochim. Acta 30(Part A), 463-477 (1974).

27 Whiffen, D. H. Infra-red summation bands of the out-of-plane $\mathrm{C}-\mathrm{H}$ bending vibrations of substituted benzene compounds. Spectrochim. Acta 7, 253-263 (1955).

28 Saito, H. \& Inoue, T. Chain orientation and intrinsic anisotropy in birefringence-free polymer blends. J. Polym. Sci. Part B Polym. Phys. 25, 1629-1636 (1987).

29 Jasse, B. \& Koenig, J. L. Orientational measurements in polymers using vibrational spectroscopy. J. Macromol. Sci. Rev. Macromol. Chem. 17, 61-135 (1979).

30 Healy, E. F. \& Holder, A. An evaluation of AM1 calculated vibrational frequencies. J. Mol. Struct. (Theochem). 281, 141-156 (1993).

31 Kurtz, H. A., Stewart, J. J. P. \& Dieter, K. M. Calculation of the nonlinear optical properties of molecules. J. Comput. Chem. 11, 82-87 (1990).

32 Turi, E. A. Thermal Characterization of Polymeric Materials 2nd edn, Vol. 1, 507 (Academic Press, San Diego, CA, 1997).

33 Rogers, S. \& Mandelkern, L. Glass transitions of the poly-(n-alkyl methacrylates). J. Phys. Chem. 61, 985-991 (1957).

34 Yazdani-Peddram, M., Soto, E., Tagle, L. H., Diaz, F. R., Gargallo, L. \& Radiæ, D. Effect of the side chain structure on the glass transition temperatures of some poly(thiocarbonate)S. Thermochim. Acta 105, 149-160 (1986). 